\title{
Bilateral Chorioretinal Folds in Association with Chronic Polypoidal Choroidal Vasculopathy
}

\begin{abstract}
Keywords: Polypoidal choroidal vasculopathy; Chorioretinal folds Retinochoroidal folds; Choroid; Choroidal folds; Macular degeneration
\end{abstract}

Chorioretinal folds have been associated with a myriad of conditions including axial hyperopia, hypotony, uveal effusion, idiopathic intracranial hypertension, orbital tumors, central serous chorioretinopathy, and posterior scleritis [1-3]. As mechanical factors and volume constraint are responsible for the anatomic distortions seen in very short eyes, the condition in general represents an anatomic perturbation of the choroid. Since it is a highly vascular structure, one might expect to find associated abnormalities and characteristics of the impacted blood vessels of the choriocapillaris. Polypoidal choroidal vasculopathy (PCV) is by far the most common form of exudative macular degeneration in blacks and Asians [4]. We describe a case of bilateral idiopathic chorioretinal folds in a Caribbean-female with associated PCV.

\section{Case Report}

A 72-year old Black-Haitian woman was seen in November of 2010 for a routine follow up retinal examination. A solitary polypoidal subretinal neovascular complex was noted in the left eye (Figures
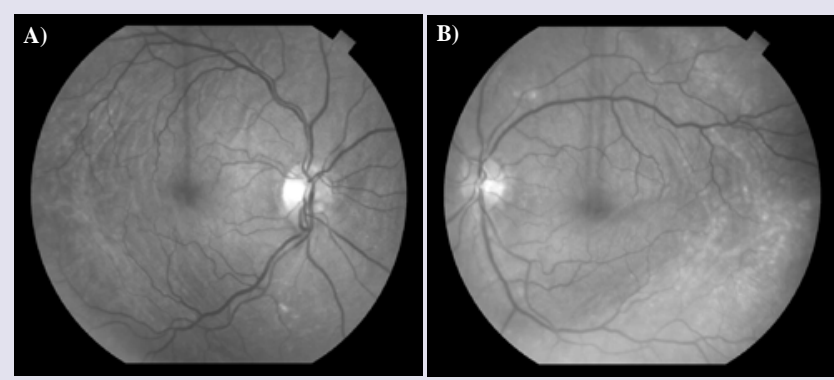

Figure 1: Red-free photographs of right and left eyes (August, 2010). Large drusen and chorioretinal folds are evident.

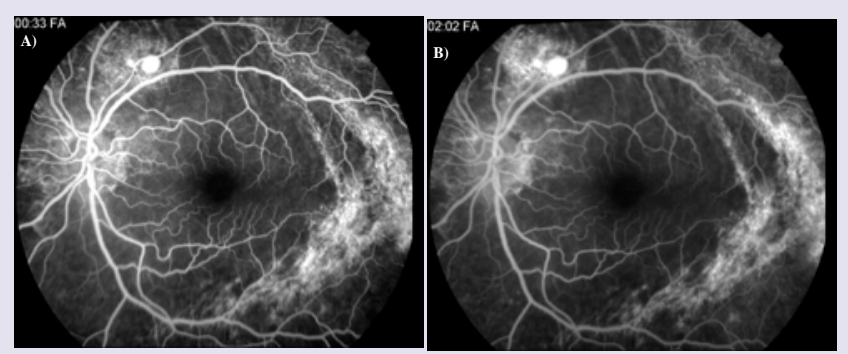

Figure 2: Early and mid-phase Fluorescein angiography of the left eye displays hyperfluorescence corresponding to choroidal neovascularization and window defect from longstanding choroidal folds (August, 2010).

\section{Journal of Ocular Biology}

\section{Eric M. Shrier* and James F. McGroarty}

Department of Ophthalmology, SUNY-Downstate Medical Center, Brooklyn, NY 11203, USA

*Address for Correspondence

Eric M. Shrier, DO, Assistant Professor and Director of Ophthalmology, Retina Service, Department of Ophthalmology, Box \#58, SUNYDownstate Medical Center, 142 Joralemon Street, Brooklyn, NY 11203, USA, Tel: 718-596-6800; E-mail: eshrier@yahoo.com

Copyright: () 2013 Shrier EM, et al. This is an open access article distributed under the Creative Commons Attribution License, which permits unrestricted use, distribution, and reproduction in any medium, provided the original work is properly cited.

Submission: 12 August 2013

Accepted: 16 September 2013

Published: 20 September 2013

Reviewed \& Approved by: Nabil M. Jabbour

Vitreous \& Retina Service

West Virginia University Eye Institute, USA

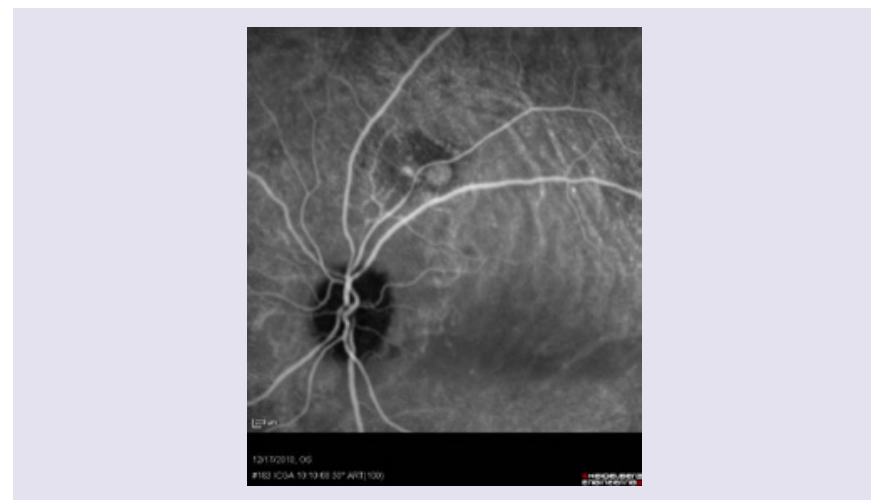

Figure 3: ICG image from left eye shows prominent staining of polypoidal neovascular complex (December, 2010).

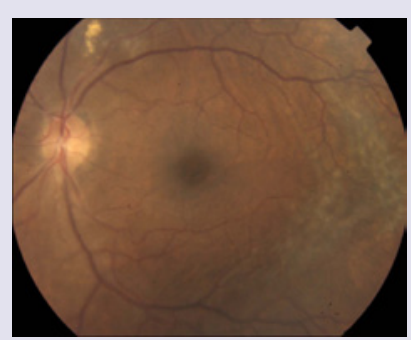

Figure 4: Color fundus photographs of the left eye (September, 2005) Drusen, chorioretinal folds and the polypoidal complex barely visible above the supero-temporal arcade.

1-3). She had been first imaged in 2005 where a diagnosis of bilateral chronic idiopathic chorioretinal folds and bilateral drusen/nonexudative macular degeneration was made (Figure 4). Her medical history was notable for treated- hypertension, hypercholesterolemia, asthma and breast carcinoma currently in remission (had been surgically treated in 1979 and 1991). She specifically denied any history of eye pain, headaches, visual loss or diplopia. Thorough review of systems was otherwise non-contributory. She denies prior history of endocrinologic or thyroid disease and has never smoked. The optic nerves have never appeared edematous on examination and her intraocular pressure has always been within a relatively 
normal range (> $14 \mathrm{~mm}$ but less than $22 \mathrm{~mm} \mathrm{Hg}$ ). She had been rendered bilaterally pseudophakic in 2005 , via uncomplicated smallincision phacoemulsification surgery, prior to which time she was not significantly hyperopic. She denied wearing spectacles during childhood. Her axial length is $22 \mathrm{~mm}$ o.u. Her best-corrected visual acuity had been stable for several years at approximately $20 / 40$ o.u.

\section{Discussion}

Bilateral chorioretinal folds are uncommon, and have been associated with choroidal venous congestion [4]. Mechanical factors such as scleral thickening may possibly predispose to derangement of the underlying circulation and theoretically to the development of subretinal neovascularization, such as occurred in this case. The biomechanical effect of scleral thickening or relative foreshortening of the eye as is the case in hyperopia is presupposed to lead to folds of the overlying choriocapillaris and retina [5], as is also intuitively the cause of (mechanical) folds that are seen in situations where there are compressive orbital tumors.

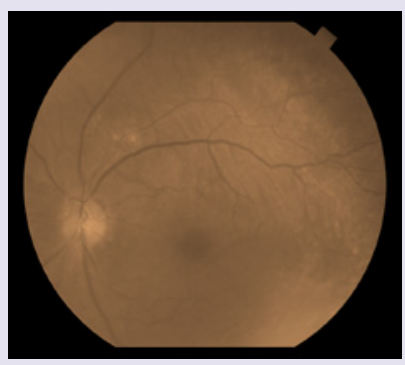

Figure 5: Color fundus photo of left eye (August, 2010). Minimal interval change of polypoidal complex versus that of 2005 (see Figure 4).
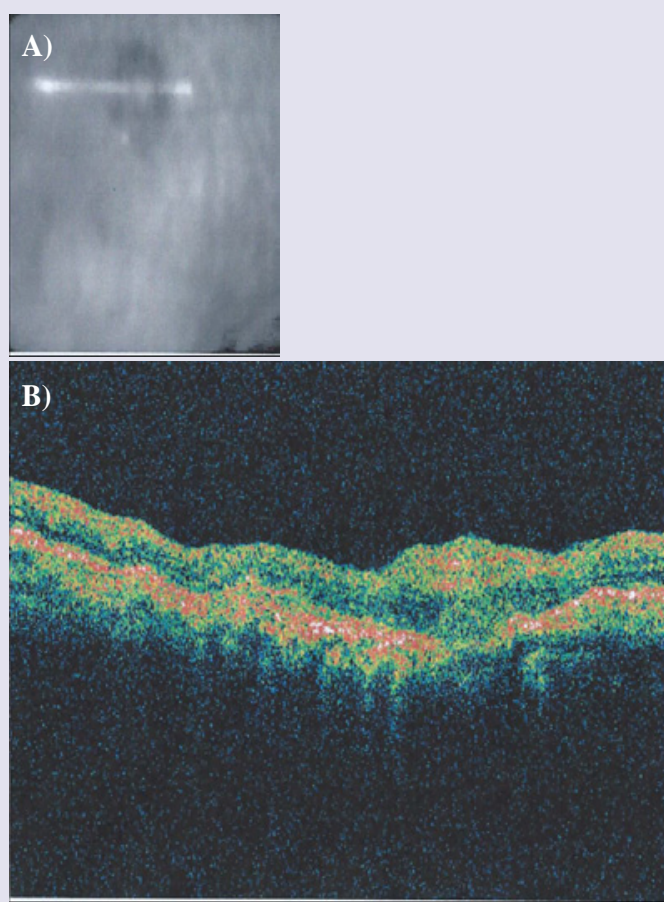

Figure 6: Reference box (a) and Time-domain OCT (Topcon III) image (b) through area of prior laser treatment o.s. Deep layer hyper-reflectivity is seen which corresponds to hyper-pigmentation and a nodular area is depicted at the location of the prior PCV polyp.

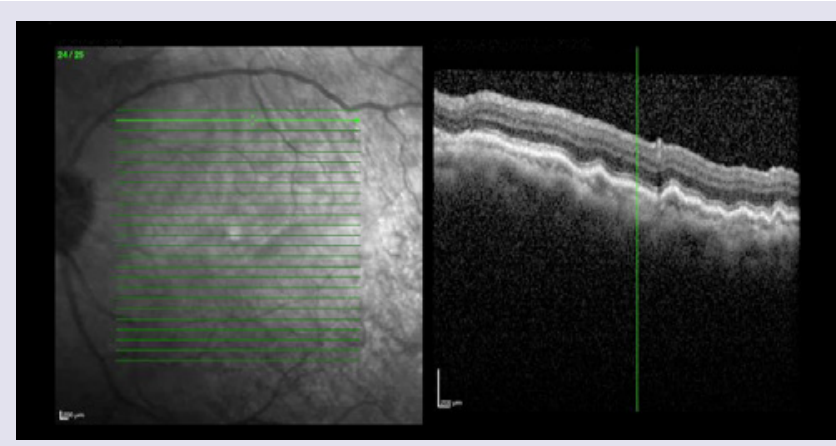

Figure 7: Spectral-domain OCT o.s. (Heidelberg Spectralis) image depicting chorioretinal folds (2011)

Rarely, choroidal folds have been reported in the setting of exudative age-related macular degeneration, but the folds in these cases were very localized and corresponded to pigment epithelial detachment and occult subretinal neovascularization [6-8]. The extensive, peculiar hyperpigmentation seen bilaterally and symmetrically in this case appeared to correspond to areas of very longstanding chorioretinal (CR) folds, as they seem to be concentric to location of existing folds.

To our knowledge, this is the first case of bilateral idiopathic chorioretinal (CR) folds seen in association with PCV. The appearance and hyper-pigmentation in the setting of the CR folds is quite striking. Given the concomitant significant hypertrophic pigmentary changes in this case, it is possible that PCV may have been underappreciated initially. It could also be easily missed in a similar setting. When seen in association with CR folds, the PCV may as in this case tend to be very indolent and remain stable over the course of time. Fluorescein and Indocyanine - Green angiography can be performed in cases of chronic chorioretinal folds especially where significant hyperpigmentation is present to aid in early detection of small, potentially hidden, polypoidal neovascular complexes. OCT also appears to well-exhibit the presence and extent of CR folds (Figures 6 and 7).

\section{References}

1. Steuhl KP, Richard G, Weidle EG (1985) Clinical observations concerning choroidal folds. Ophthalmologica 190: 219-224.

2. Griebel SR, Kosmorsky GS (2000) Choroidal folds associated with increased intracranial pressure. Am J Ophthalmol 129: 513-516.

3. Sarraf D, Schwartz SD (2003) Bilateral choroidal folds and optic neuropathy a variant of the crowded disk syndrome? Ophthalmology 110: 1047-1052.

4. Yannuzzi LA (2010) The Retinal Atlas. Saunders.

5. Friberg TR (1989) The etiology of choroidal folds. A biomechanical explanation. Graefes Arch Clin Exp Ophthalmol 227: 459-464.

6. Haruyama M, Yuzawa M, Kawamura A, Yamazaki C, Matsumoto Y (2001) Indocyanine green angiographic findings of chorioretinal folds. Jpn J Ophthalmol 45: 293-300.

7. Cohen SM, Gass JD (1994-1995) Bilateral radial chorioretinal folds. Int Ophthalmol 18: 243-245.

8. Menard C, Cohen SY, Perrenoud F, Coscas G (1992) Idiopathic and secondary chorioretinal folds. J Fr Ophthalmol 15: 497-502.

Acknowledgements

Dr. David Soltanpour, MD for performing confirmatory A-scans. 